Research/Technical Note

\title{
Streamlining the Workflow of Stereotactic Radiosurgery (SRS) on Tomotherapy: Experience from a Tertiary Care Centre from India
}

\author{
Vijay Palwe ${ }^{1,}$, , Prakash Pandit ${ }^{1}$, Rajnish Nagarkar ${ }^{2}$ \\ ${ }^{1}$ Department of Radiation Oncology, HCG Manavata Cancer Centre, Nashik, India \\ ${ }^{2}$ Department of Surgical Oncology, HCG Manavata Cancer Centre, Nashik, India
}

Email address:

academics@manavatacancercentre.com (V.Palwe),prakash@manavatacancercentre.com (P. Pandit)

${ }^{*}$ Corresponding author

To cite this article:

Vijay Palwe, Prakash Pandit, Rajnish Nagarkar. Streamlining the Workflow of Stereotactic Radiosurgery (SRS) on Tomotherapy: Experience from a Tertiary Care Centre from India. Radiation Science and Technology. Vol. 5, No. 1, 2019, pp. 1-4. doi: 10.11648/j.rst.20190501.11

Received: February 18, 2019; Accepted: March 30, 2019; Published: June 4, 2019

\begin{abstract}
Brain metastasis has become a major concern in the oncology fraternity. The use of conventional treatment approaches such as single-fraction stereotactic radiosurgery (SRS), hypofractionated stereotactic radiotherapy (SRT), or whole-brain radiotherapy (WBRT) has been widely explored. Stereotactic radiosurgery (SRS) has been widely used and known for its high efficiency and low toxicity. SRS on tomotherapy has emerged as a promising treatment approach at our centre. In the advent of multidisciplinary care, developing and implementing targeted treatment approaches for cancer patients, SRS has proven to be highly effective and efficient. We report our first experience of brain metastases in a known case of breast cancer treated with SRS on tomotherapy in a tertiary cancer centre in India.
\end{abstract}

Keywords: Stereotactic Radiotherapy, Tomotherapy, Brain Metastasis, Stereotactic Radiosurgery Component

\section{Introduction}

Cancer patients presenting with brain metastasis has becoming a growing concern. The conventional method for treating such patients involved single-fraction stereotactic radiosurgery (SRS), hypofractionated stereotactic radiotherapy (SRT), or whole-brain radiotherapy (WBRT) [1]. Stereotactic radiosurgery (SRS) has been used for several decades in the treatment of brain metastases. It has been an ideal treatment approach considering its efficiency and effectiveness in achieving high rates of local control. In limited settings, SRS has proven to improve survival [2]. We report a case brain metastasis in a patient with previously diagnosed breast cancer treated with SRS on tomotherapy in our centre. Streamlining the process of SRS on tomotherapy was an achievement for a tertiary cancer centre based in a tier-2 city in India.

\section{Case Presentation}

A 36-year-old female presented to our clinic with complaints of difficulty in reading and writing. The patient was a primary teacher by profession. She was a known case of breast cancer. She was diagnosed with triple negative carcinoma of the left breast in October 2015. The patient had completed her entire treatment course that included breast conservation therapy/surgery (BCT), adjuvant chemotherapy (four cycles of doxorubicin and cyclophosphamide) and 12 cycles weekly paclitaxel. The patient was also given adjuvant external radiotherapy till June 2016.

The patient was on regular follow-up. The contrast MRI brain showed a solitary space occupying lesion (SOL) in the left high parietal region (Figure 2). The whole body Positron Emission Tomography/Computed Tomography (PET-CT) scan showed no metastasis in the body.

Post discussion with our multidisciplinary team and the patient, it was decided to plan her for stereotactic radiosurgery 
(SRS) on tomotherapy.

The patient was planned with CT simulation and MRI on the first day followed by treatment on the consecutive day.

The clinical procedure for SRS would be streamlined that would typically follow the Intensity-Modulated Radiation Therapy (IMRT) procedure. The procedure would be as follows: (a) Immobilization and CT-stimulation (b) CT-MRI fusion (c) Treatment Planning and (d) Dose delivery.

\section{Helical Tomotherapy (HT)}

The helical tomotherapy unit is a system consisting of a small $6 \mathrm{MV}$ linear accelerator mounted on a ring gantry that rotates isocentrically around the patient (Figure 1). The patient receives the radiation dose through a helical path as he/she moves through the bore. The beam intensity is modulated using a binary collimator from the inside.

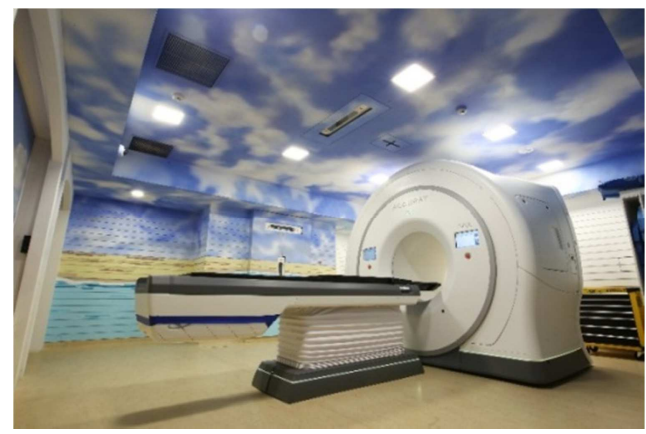

Figure 1. Helical Tomotherapy unit at HCG Manavata Cancer Centre.

The binary collimator has two banks with each having 64 leaves with a beamlet size of $0.625 \mathrm{~cm}$. The collimator gives a total field width of $40 \mathrm{~cm}$.

The Helical Tomotherapy unit also has MVCT imaging that helps provide image-guided radiation therapy (IGRT) capability for accurate patient setup. The patient's head is immobilized with the help of a specially designed thermoplastic mask system. The process is performed prior to CT simulation.

During the fabrication process, the thermoplastic mask is pulled over in a manner that accommodates the patient's face. The next step involves taking a CT image dataset at $1.25 \mathrm{~mm}$ slice thickness from the top of the head through the cervical spine.
Post-CT scan, the patient is taken for MRI scan in the treatment position. Both the CT and MRI image datasets are transferred to the Eclipse Planning System. The system helps in determining target volume, organs at risk volumes, and pseudo-structures contouring.

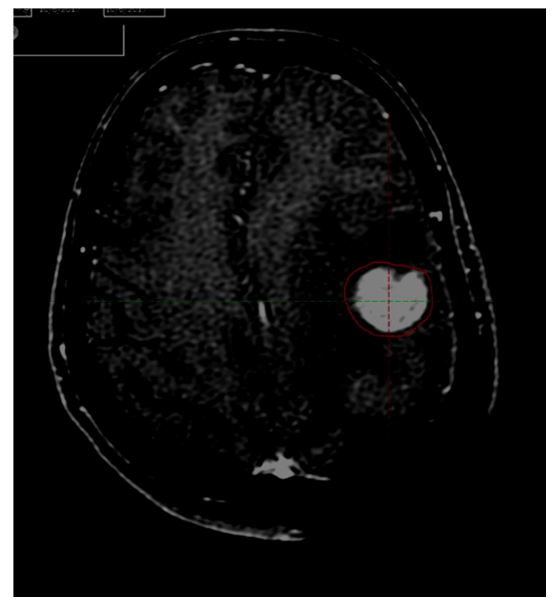

Figure 2. MRI Brain showing left high parietal space occupying lesion (SOL).

The structure and image dataset are exported to the TomoHD treatment planning system. The treatment plan is evaluated to warrant that the plan meets the target conformity index and normal tissue dose-volume constraints (Figure 3). Post-acceptance of the treatment plan, a delivery quality assurance (DQA) plan is generated.

The patient is set up on the Helical TomoTherapy unit on the day of the treatment based on the parameters on the device on the day of the CT-simulation. The machine-based treatment parameters are downloaded and an image-guided procedure is performed. The process involves acquisition of MVCT image dataset at the fine level ( $2 \mathrm{~mm}$ slice). It is co-registered against the CT-simulation image dataset. Co-registration is done on the bony structures of the skull with focus near the treatment region by the radiation therapist and approved by the radiation oncologist. Post approval from the team, the treatment commences. Another MVCT image dataset is collected after the first treatment sessions is completed. Co-registration is performed to evaluate and confirm the patient position. A one-month follow-up showed good response on MRI post-SRS (Figuer 4). Figure is as follows:

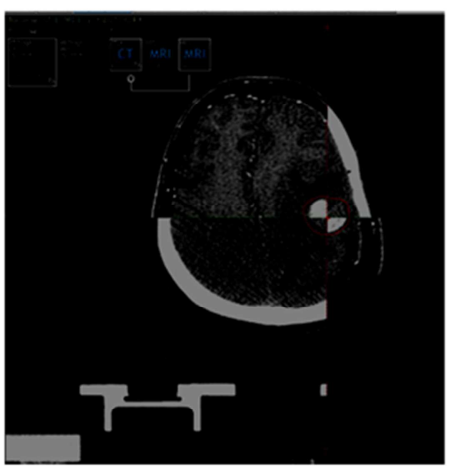

(a) CT-MRI Fusion

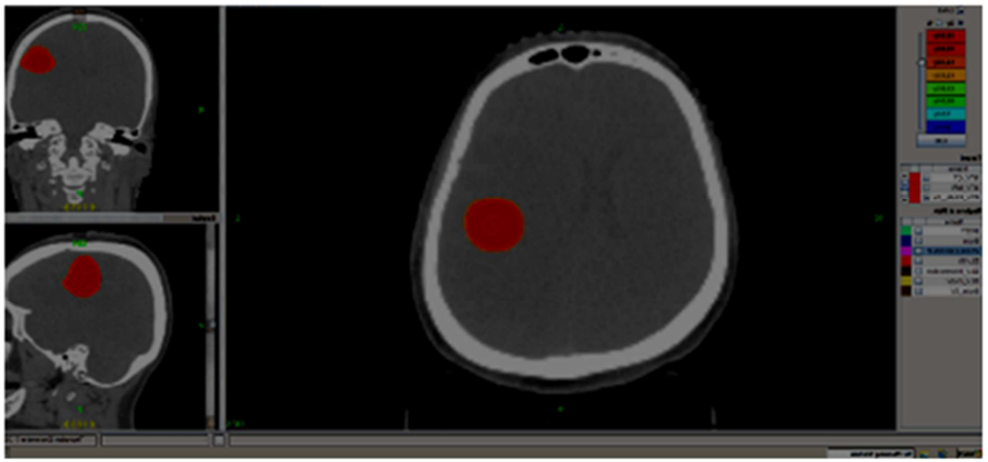

(b) Dosing in tomotherapy plan

Figure 3. Tomotherapy Plan 


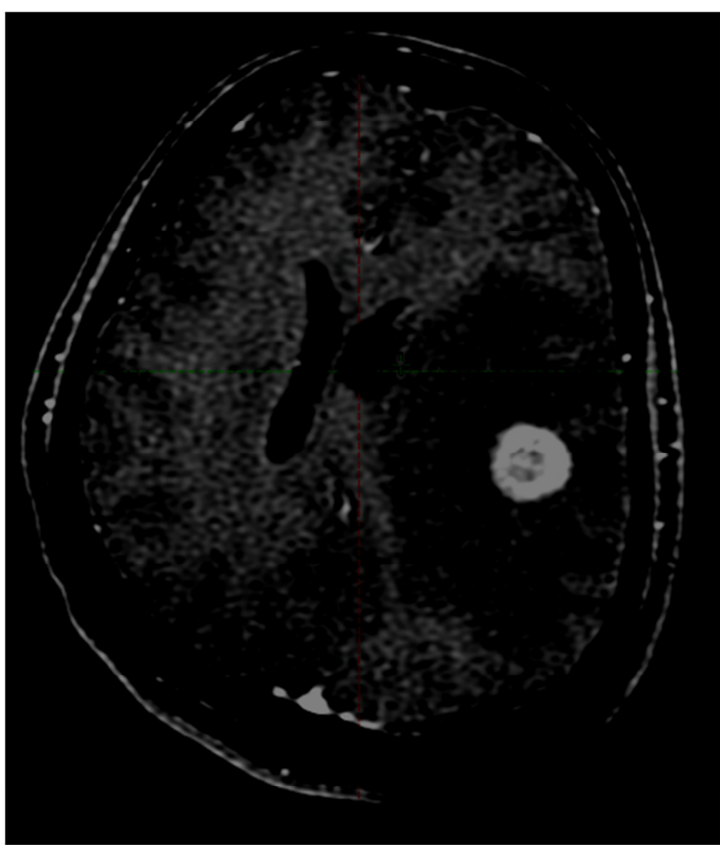

(a) Pre-SRS MRI

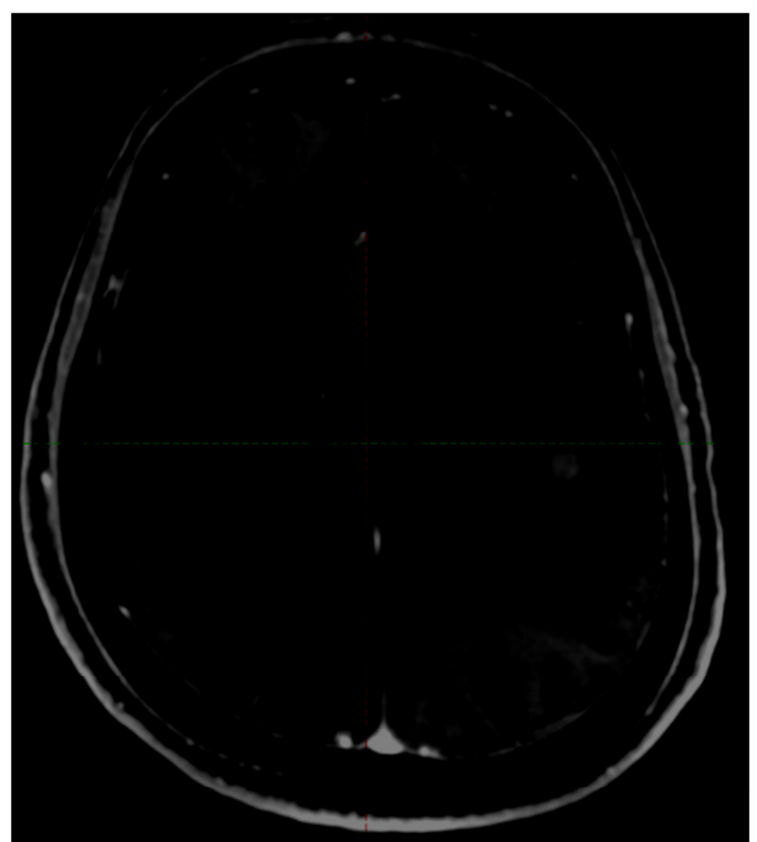

(b) Post-SRS MRI

Figure 4. Pre-treatment and Post-SRS MRI Scan comparison (One-month follow-up).

\section{Discussion}

Metastatic brain tumors comprise of nearly $20-40 \%$ of all patients diagnosed with cancer [3-4]. As per current evidence, the primary treatment approaches for metastatic brain tumors include surgery, whole brain radiation therapy (WBRT), and stereotactic radiosurgery (SRS) [3-5]. WBRT has remained the primary treatment for patients presenting with high intracranial tumor burden. However, the routine use of WBRT as an adjunct therapy for patients who are candidates for surgical resection or SRS has changed. No known survival benefit with WBRT has been reported. Furthermore, WBRT is known to have a detrimental impact on the patient's quality of life. Patients who undergo WBRT often suffer from a neurocognitive decline [6-7].

As per a consensus of national radiosurgical and radiation oncological societies, SRS alone has been recommended for patients with 4 or less lesions $[8,9]$.

The Helical TomoTherapy unit has a unique hardware, i.e. the helical motion which helps deliver radiation dose continuously around the patient combined with the MVCT image-guided system. The same TomoHD treatment planning system is also used for focal irradiation and IMRT. The patient is discharged immediately after completion of the CT-simulation and thin slice MRI scan. The patient is called on the treatment day after planning is complete. A standard patient-specific quality assurance (QA) for IMRT patients have been used for the SRS patients. The overall workflow process for radiosurgery is similar to IMRT. SRS treatment using Helical Tomotherapy is no longer a whole day even at it used to be with other dose delivery systems. It is similar to that of the IMRT procedure.

In the era of novel and effective 'targeted therapy' such as cellular and molecular-oriented therapies', SRS has emerged as an evolving approach in multidisciplinary care for cancer patients [10]. As per current evidence, the use of SRS as an adjunct or as a single treatment modality for brain metastases has gained momentum. SRS has been found to be a highly effective treatment modality for the treatment of brain metastases [11]. The main advantage of SRS is that it is a minimally invasive procedure which is highly effective against spherical and well-demarcated characteristics of most brain metastases [12]. Some of the other benefits of SRS include few side-effects, single session delivery of high-dose radiation, and a minimal delay to systemic therapy [13]. SRS has been proven to have excellent local control with low toxicity in highly selected breast cancer patients [14]. Tomotherapy has been presented as a precise SRS delivery system known for its accuracy and efficiency [15].

\section{Conclusion}

The implementation of stereotactic radiosurgery on the Helical Tomotherapy can be streamlined well at our tertiary care centre. The only exception is the thin slice planning MRI scan fusion of the dataset with the same thin sliced planning CT images. The overall process and clinical setup is similar to the routine clinical practice for IMRT patients. The treatment time for SRS on Helical tomotherapy is short, i.e. 10-15 minutes which is convenient and safe for patients.

\section{Acknowledgements}

We would like to thank Mr. Lyndon Fernandes for his medical writing assistance. 


\section{References}

[1] Nagai A, Shibamoto Y, Yoshida M, Wakamatsu K, Kikuchi Y. Treatment of single or multiple brain metastases by hypofractionated stereotactic radiotherapy using helical tomotherapy. Int J Mol Sci. 2014; 15 (4): 6910-24. Published 2014 Apr 22. doi: 10.3390/ijms15046910.

[2] Vellayappan BA, Doody J, Vandervoort E, et al. Pre-operative versus post-operative radiosurgery for brain metastasis: Effects on treatment volume and inter-observer variability. J Radiosurg SBRT. 2018; 5 (2): 89-97.

[3] Soffietti R1, Cornu P, Delattre JY, Grant R, Graus F, Grisold W, Heimans J, Hildebrand J, Hoskin P, Kalljo M, Krauseneck P, Marosi C, Siegal T, Vecht C. EFNS Guidelines on diagnosis and treatment of brain metastases: report of an EFNS Task Force. Eur J Neurol. 2006 Jul; 13 (7): 674-81.

[4] Märtens B, Janssen S, Werner M, et al. Hypofractionated stereotactic radiotherapy of limited brain metastases: a single-centre individualized treatment approach. BMC Cancer. 2012; 12: 497. doi: 10.1186/1471-2407-12-497.

[5] Nieder C; Grosu AL; Gaspar LE. Stereotactic radiosurgery (SRS) for brain metastases: a systematic review. Radiat Oncol 9: $155 ; 2014$.

[6] Gaspar L, Scott C, Rotman M, Asbell S, Phillips T, Wasserman T, McKenna WG, Byhardt R. Recursive partitioning analysis (RPA) of prognostic factors in three Radiation Therapy Oncology Group (RTOG) brain metastases trials. Int J Radiat Oncol Biol Phys. 1997 Mar 1; 37 (4): 745-51.

[7] Borgelt B, Gelber R, Kramer S, Brady LW, Chang CH, Davis LW, Perez CA, Hendrickson FR. The palliation of brain metastases: final results of the first two studies by the Radiation
Therapy Oncology Group. Int J Radiat Oncol Biol Phys. 1980 Jan; 6 (1): 1-9.

[8] Khan A; Dicker A. On the merits and limitations of whole brain therapy. JCO 31: 11-13; 2013.

[9] Sahgal A, Aoyama H, Kocher M, Neupane B, Collette S, Tago M, Shaw P, Beyene J, Chang EL. Phase 3 trials of stereotactic radiosurgery with or without whole-brain radiation therapy for 1 to 4 brain metastases: individual patient data meta-analysis. Int J Radiat Oncol Biol Phys. 2015 Mar 15; 91 (4): 710-7.

[10] Zeng M, Han LF. Stereotactic radiosurgery: a "targeted" therapy for cancer. Chin J Cancer. 2012; 31 (10): 471-5.

[11] Cohen-Inbar O, Sheehan JP. The role of stereotactic radiosurgery and whole brain radiation therapy as primary treatment in the treatment of patients with brain oligometastases - A systematic review. J Radiosurg SBRT. 2016; 4 (2): 79-88.

[12] Yaeh A, Nanda T, Jani A, Rozenblat T, Qureshi Y, Saad S, Lesser J, Lassman AB, Isaacson SR, Sisti MB, Bruce JN, McKhann GM 2nd, Wang TJ. Control of brain metastases from radioresistant tumors treated by stereotactic radiosurgery. J Neurooncol. 2015 Sep; 124 (3): 507-14. doi: 10.1007/s11060-015-1871-5.

[13] Soffietti R, Rudā R, Mutani R. Management of brain metastases. J Neurol. 2002 Oct; 249 (10): 1357-69.

[14] Kirova YM, Chargari C, Zefkili S, Campana F. Could helical tomotherapy do whole brain radiotherapy and radiosurgery? World J Radiol. 2010; 2 (4): 148-50.

[15] Soisson ET, Hoban PW, Kammeyer T, et al. A technique for stereotactic radiosurgery treatment planning with helical tomotherapy. Med Dosim. 2011; 36 (1): 46-56. 